\title{
A massive protostellar core with an infalling envelope ${ }^{\star}$
}

\author{
S. M. Birkmann ${ }^{1}$, O. Krause ${ }^{1}$, M. Hennemann ${ }^{1}$, Th. Henning ${ }^{1}$, J. Steinacker ${ }^{1,2}$, and D. Lemke ${ }^{1}$ \\ 1 Max-Planck-Institut für Astronomie (MPIA), Königstuhl 17, 69117 Heidelberg, Germany \\ e-mail: [birkmann; krause; hennemann; henning; stein; lemke] @mpia. de \\ 2 Zentrum für Astronomie der Universität Heidelberg (ZAH), Mönchhofstr. 12-14, 69120 Heidelberg, Germany
}

Received 30 January 2007 / Accepted 18 August 2007

ABSTRACT

\begin{abstract}
Context. Due to the short timescales involved and observational difficulties, our knowledge of the earliest phases of massive star formation remains incomplete.

Aims. We aim to explore the physical conditions during the initial phases of high-mass star formation and to detect a genuine massive (mass $M>8 M_{\odot}$ ) protostar at an early evolutionary stage.

Methods. We have launched a multi-wavelength study of young and massive star-forming regions that were identified by the ISOPHOT Serendipity Survey (ISOSS) performed with the ISO space telescope. The follow-up observations include ground-based near-infrared imaging and (sub)mm continuum and molecular line measurements (both single-dish and interferometric), as well as mid- to far-infrared measurements with the Spitzer Space Telescope. The combined spectrophotometric data are used to determine source temperatures $T$ and masses $M$.

Results. ISOSS J23053+5953 is a massive $\left(M \sim 900 M_{\odot}\right.$, luminosity $\left.L \sim 2100 L_{\odot}\right)$ and cold $(T \sim 17 \mathrm{~K})$ star-forming region with two protostellar/protocluster candidates $\left(T \lesssim 20 \mathrm{~K}\right.$ and $T \sim 17.5 \mathrm{~K}, M \sim 200 M_{\odot}$ each). The low temperatures are strongly confined by the spectrophotometric Spitzer data in the FIR. Interferometric observations reveal that the colder core (SMM2) has a mass of $M=26 M_{\odot}$ within a region of $8700 \times 5600$ AU and drives an outflow. It also shows signs of infall in both single-dish and interferometric measurements, and its luminosity can be explained by accretion. We also detect a large-scale jet that is traced by $\mathrm{H}_{2}$ emission. Conclusions. The cold mm-core ISOSS J23053+5953 SMM2 is a promising candidate for a high-mass protostar in an early evolutionary stage and one of the few objects showing both infall signatures and jets as a sign of accretion.
\end{abstract}

Key words. ISM: individual objects: ISOSS J23053+5953 - stars: formation - ISM: jets and outflows - infrared: ISM ISM: kinematics and dynamics

\section{Introduction}

Recently, significant observational evidence has been found that the accretion scenario is also valid in the regime of high-mass star formation (Beltrán et al. 2006; Beuther et al. 2006; Beuther \& Steinacker 2007; Puga et al. 2006; Keto \& Wood 2006; Steinacker et al. 2006). This poses the question of whether the initial conditions under which massive stars form are also comparable to low-mass star birth, i.e. are characterized by cold $(T<10 \mathrm{~K})$ prestellar cores (Ward-Thompson 2002). Several promising candidates for very young high-mass protostars have been discovered near more evolved luminous objects (Garay et al. 2004; Forbrich et al. 2004; Wu et al. 2005; Beuther et al. 2005), but no clear example of a massive prestellar core is known so far.

We have used the $170 \mu \mathrm{m}$ ISOPHOT Serendipity Survey (ISOSS) (Lemke et al. 1996; Bogun et al. 1996) carried out during the ISO mission (Kessler et al. 1996) to search for extremely young and massive star-forming regions. To explore the physical conditions in this unique sample of cold and massive star-forming regions and to search for possible highmass prestellar cores, we have launched a multi-wavelength follow-up survey (Krause et al. 2003; Birkmann et al. 2006).

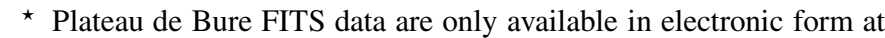
the CDS via anonymous ftp to

cdsarc.u-strasbg.fr $(130.79 .128 .5)$ or via

http://cdsweb.u-strasbg.fr/cgi-bin/qcat? J/A+A/474/883
The data include near-infrared imaging, single-dish and interferometric molecular-line and continuum observations in the (sub)millimeter regime, and mid- to far-infrared observations with the Spitzer Space Telescope. In this paper we present our findings on cold cores in the star-forming region ISOSS $\mathrm{J} 23053+5953$, placed towards the Cepheus molecular cloud complex at a distance of $\sim 3.5 \mathrm{kpc}$ (Wouterloot et al. 1989, and references therein).

\section{Observations and data reduction}

ISOSS J23053+5953 coincides with IRAS 23032+5937; $\operatorname{RA}(2000)=23^{\mathrm{h}} 05^{\mathrm{m}} 23^{\mathrm{s}} \cdot 1$, Dec $\left.(2000)=+59^{\circ} 53^{\prime} 52^{\prime \prime}\right)$. A $170 \mu \mathrm{m}$ flux $F_{170 \mu \mathrm{m}}=96_{-20}^{+90}$ Jy was derived by two-dimensional Gaussian fitting of the source in the ISOSS scan map (ISOSS beam $\approx 90^{\prime \prime} F W H M$ ). A detailed description of the procedures is given by Krause (2003) and Stickel et al. (2004).

Sub-mm continuum jiggle maps at $850 \mu \mathrm{m}$ and $450 \mu \mathrm{m}$ were obtained with SCUBA (Holland et al. 1999) at the James Clerk Maxwell Telescope (JCMT) in May 2003 under good atmospheric transmission conditions $\left(\tau_{850 \mu \mathrm{m}} \sim 0.2\right)$. The photometric calibration is based on maps of Uranus acquired directly before the observations with an estimated accuracy of $20 \%$ at $450 \mu \mathrm{m}$ and $12 \%$ at $850 \mu \mathrm{m}$. The $F W H M$ beam size is $7.5^{\prime \prime}$ and $14.5^{\prime \prime}$, respectively.

Near-infrared images in $J, H, K \mathrm{~s}$, and in the $v=1-0 \mathrm{~S}(1)$ line $(\lambda=2.122 \mu \mathrm{m})$ of molecular hydrogen have been 
Table 1. Observed and detected molecular lines presented in this paper.

\begin{tabular}{|c|c|c|c|c|c|c|c|c|}
\hline $\begin{array}{c}\text { Molecular } \\
\text { line }\end{array}$ & Transition & $\begin{array}{c}\text { Frequency } \\
{[\mathrm{GHz}]}\end{array}$ & $\begin{array}{c}\text { Date } \\
\text { observed }\end{array}$ & Telescope & $\begin{array}{l}\text { Beam } \\
\text { size }\end{array}$ & $\tau_{230 \mathrm{GHz}}$ & $\begin{array}{l}T_{\text {sys }} \\
{[\mathrm{K}]}\end{array}$ & $\begin{array}{c}\text { Peak } T_{\mathrm{MB}} \\
{[\mathrm{K}]}\end{array}$ \\
\hline $\mathrm{HCO}^{+}$ & $J=3 \rightarrow 2$ & 267.5576 & \multirow[t]{2}{*}{ November 62003} & \multirow[t]{2}{*}{ IRAM $30 \mathrm{~m}$} & $9.5^{\prime \prime}$ & $\sim 0.45$ & $\sim 1600-1800$ & 7.5 \\
\hline $\mathrm{H}^{13} \mathrm{CO}^{+}$ & $J=3 \rightarrow 2$ & 260.2555 & & & $9.5^{\prime \prime}$ & $\sim 0.45$ & $\sim 1700$ & 1.2 \\
\hline $\mathrm{CO}$ & $J=3 \rightarrow 2$ & 345.7960 & December 182004 & \multirow[t]{6}{*}{ HНT 10 m } & $22^{\prime \prime}$ & $\sim 0.12$ & $\sim 600$ & 9.5 \\
\hline $\mathrm{C}^{18} \mathrm{O}$ & $J=2 \rightarrow 1$ & 219.5604 & January 162006 & & $34^{\prime \prime}$ & $\sim 0.26$ & $\sim 700$ & 0.8 \\
\hline $\mathrm{H}_{2} \mathrm{CO}$ & $3_{03} \rightarrow 2_{02}$ & 218.2222 & January 182006 & & $35^{\prime \prime}$ & $\sim 0.21$ & $\sim 630$ & 0.75 \\
\hline \multirow[t]{3}{*}{$\mathrm{CH}_{3} \mathrm{OH}$} & $5_{0} \rightarrow 4_{0}-E$ & 241.7002 & \multirow[t]{3}{*}{ January 192006} & & $31^{\prime \prime}$ & $\sim 0.16$ & $\sim 530$ & 1.26 \\
\hline & $5_{-1} \rightarrow 4_{-1}-E$ & 241.7672 & & & $31^{\prime \prime}$ & & & 1.05 \\
\hline & $5_{0} \rightarrow 4_{0}-A$ & 241.7914 & & & $31^{\prime \prime}$ & & & 0.29 \\
\hline $\mathrm{CO}$ & $J=2 \rightarrow 1$ & 230.5380 & January to & \multirow[t]{4}{*}{ PdBI } & $1.15^{\prime \prime} \times 0.87^{\prime \prime}$ & $\sim 0.2$ & $\sim 400$ & $\sim 18$ \\
\hline $1.3 \mathrm{~mm}$ & continuum & 230.5 & March 2005 & & $\left(\mathrm{PA}=75^{\circ}\right)$ & & & \\
\hline $\mathrm{HCO}^{+}$ & $J=1 \rightarrow 0$ & 89.1880 & & & $2.58^{\prime \prime} \times 2.16^{\prime \prime}$ & & $\sim 150$ & $\sim 2.6$ \\
\hline $3 \mathrm{~mm}$ & continuum & 89.2 & & & $\left(\mathrm{PA}=78^{\circ}\right)$ & & & \\
\hline
\end{tabular}

obtained at the Calar Alto $3.5 \mathrm{~m}$ telescope with the two primefocus wide-field cameras Omega2000 (Baumeister et al. 2003) and OmegaPrime (Bizenberger et al. 1998). Omega2000 features a field of view (FOV) of $15.4 \times 15.4 \mathrm{arcmin}^{2}$ with a pixel scale of $0.4496^{\prime \prime}$ pix $^{-1}$, while the FOV for OmegaPrime is $6.8 \times$ $6.8 \operatorname{arcmin}^{2}$ with a pixel scale of $\sim 0.4^{\prime \prime} \mathrm{pix}^{-1}$. The exposure time in the broad band filters was $20 \mathrm{~min}$ each and $40 \mathrm{~min}$ for the narrow band filter. The exposures were dithered on source to allow for sky subtraction. Photometric calibration for $J, H$, and $K \mathrm{~s}$ is based on the 2MASS point source catalog.

The Spitzer (Werner et al. 2004) observations include IRAC (Fazio et al. 2004) imaging in all four photometric bands, MIPS (Rieke et al. 2004) imaging at $24 \mu \mathrm{m}$ and $70 \mu \mathrm{m}$, and the MIPS spectral energy distribution (SED) mode. The total exposure times per pixel were $582.4 \mathrm{~s}$ (IRAC), $312.5 \mathrm{~s}$ (MIPS $24 \mu \mathrm{m}$ ), $226.5 \mathrm{~s}$ (MIPS $70 \mu \mathrm{m}$ ), and $2 \times 189 \mathrm{~s}$ (MIPS SED, two positions). For all imaging observations we used the basic flux calibrated data (BCD) of the Spitzer Science Center (SSC) pipeline as the starting point for further data reduction and analysis. The MOPEX software (Makovoz \& Marleau 2005) was used to perform cosmetic corrections and astrometric refinement. Final images were combined using scripts in IRAF. Photometry was done using aperture and PSF fitting and with the aperture corrections given in the IRAC data handbook and the SSC website ${ }^{1}$. The MIPS SED mode observations were calibrated to a spectrum of $\alpha$ Boo (Low et al. 2005) and the measured MIPS $70 \mu \mathrm{m}$ fluxes. The photometric accuracy is estimated to $2 \%$ (IRAC), 5\% (MIPS 24), and 10\% (MIPS 70 and SED).

Molecular line measurements were obtained at the IRAM $30 \mathrm{~m}$ telescope and at the Heinrich Hertz Submillimeter Telescope (HHT). In addition we obtained interferometric data at the Plateau de Bure Interferometer (PdBI) at $3 \mathrm{~mm}$ and $1.3 \mathrm{~mm}$. An overview of all molecular line and continuum observations presented in this paper is given in Table 1 . At the IRAM $30 \mathrm{~m}$ we used the single pixel A/D receivers with a spectral resolution of $40 \mathrm{kHz}$ and a bandwidth of $80 \mathrm{MHz}$. In addition we carried out a sparse map in of $\mathrm{HCO}^{+}(3-2)$ using the nine pixel Heterodyne Receiver Array (HERA, Schuster et al. 2004) in frequency-switching mode with the VESPA correlator. At the HHT, we used the SIS $345 \mathrm{GHz}$ receiver for the $\mathrm{CO}(3-2)$ observations and the new $1 \mathrm{~mm}$ single-sideband JT receiver for the other lines. In all cases the two AOS backends with a bandwidth of $1 \mathrm{GHz}$ and a nominal resolution of $\sim 920 \mathrm{kHz}$ were

\footnotetext{
${ }^{1}$ http://ssc.spitzer.caltech.edu/mips/apercorr/
}

used. We carried out on the fly maps for the CO transitions and pointed observations for the remaining lines. The PdBI observations made use of the facility receivers and backends with resolutions of $40 \mathrm{kHz}$ ( $3 \mathrm{~mm}$ lines), $160 \mathrm{kHz}$ ( $1 \mathrm{~mm}$ lines), and $2.5 \mathrm{MHz}$ (continuum, $500 \mathrm{MHz}$ bandwidth). Phase calibrators were $2200+420$ and $0059+581$, and additional amplitude calibrators were MWC349, 3C 84, 3C 273, 1928+738, 2037+511, and $0212+735$. All radio data were reduced with the GILDAS ${ }^{2}$ software.

\section{Results}

\subsection{Maps and fluxes}

The SCUBA sub-mm map of ISOSS J23053+5953 is shown in the left panel of Fig. 1. Two cores are detected in addition to a more extended emission component. With a beam size of $14.5^{\prime \prime}$ at $850 \mu \mathrm{m}$ the two cores are clearly separated but unresolved. At $450 \mu \mathrm{m}$ however (beam size 7.5'), the northeastern core (SMM1) appears extended while the southwestern core (SMM2) is unresolved. The separation between the centers of the two emission peaks is $\approx 17^{\prime \prime}$ with a position angle of $\mathrm{PA}=50^{\circ}$.

The fluxes of the two cores were derived using the methods described by Sandell \& Weintraub (2001) and are $12.8 \pm 2.6 \mathrm{Jy}$ and $1.65 \pm 0.25 \mathrm{Jy}$ for SMM1 and $10.3 \pm 2.1 \mathrm{Jy}$ and $1.51 \pm$ $0.23 \mathrm{Jy}$ for SMM2 at 450 and $850 \mu \mathrm{m}$, respectively. The flux of the entire region (aperture of $90^{\prime \prime}$ diameter) is $34 \pm 7 \mathrm{Jy}$ at $450 \mu \mathrm{m}$ and $8.3 \pm 1.2 \mathrm{Jy}$ at $850 \mu \mathrm{m}$. The contamination of the the $850 \mu \mathrm{m}$ continuum flux by $\mathrm{CO}(3-2)$ line emission deduced from our map obtained at the HHT is $\lesssim 6 \%$, well within the photometric errors.

At $70 \mu \mathrm{m}$ (middle panel of Fig. 1), the two cores are just separated with the resolution provided by MIPS $\left(\sim 18^{\prime \prime} F W H M\right)$. We used PSF fitting to derive fluxes of $F_{70 \mu \mathrm{m}}=29.5 \pm 3.0 \mathrm{Jy}$ for SMM1 and $F_{70 \mu \mathrm{m}}=11.7 \pm 1.2 \mathrm{Jy}$ for SMM2. The flux of the entire region, determined by aperture photometry, is $F_{70 \mu \mathrm{m}}=$ $44.7 \pm 4.5 \mathrm{Jy}$. Thus the two cores contribute more than $90 \%$ of the total flux measured at $70 \mu \mathrm{m}$.

The central region of our deep MIPS $24 \mu \mathrm{m}$ map is presented in the right panel of Fig. 1. Six emission maxima are detected in the vicinity of the sub-mm cores, two being located near the peak of the thermal dust continuum emission observed at $450 \mu \mathrm{m}$. We derive a flux of $F_{24 \mu \mathrm{m}}=0.77 \pm 0.04 \mathrm{Jy}$ for

\footnotetext{
${ }^{2}$ http://www.iram.fr/IRAMFR/GILDAS
} 


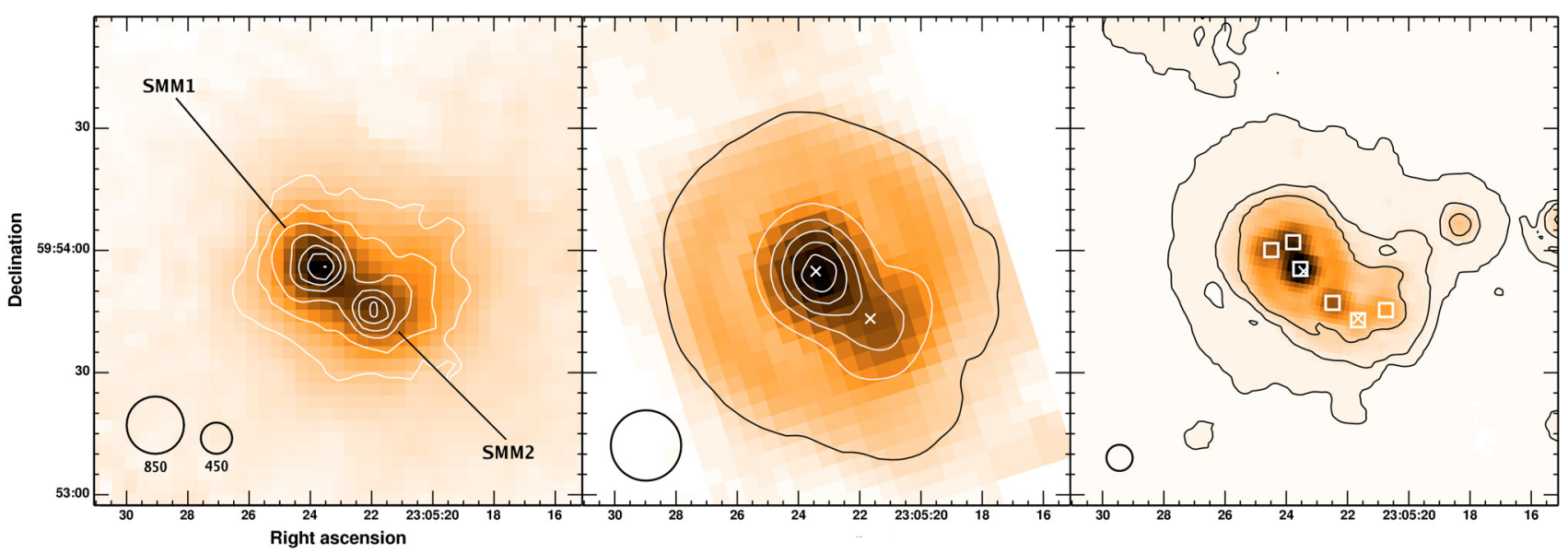

Fig. 1. Left: map of the $850 \mu \mathrm{m}$ emission of ISOSS J23053+5953 taken with SCUBA. The color scale is linear with a maximum brightness of $1.5 \mathrm{Jy}_{\text {beam }}{ }^{-1}$. The contours show the emission at $450 \mu \mathrm{m}$ in steps of $1 \mathrm{Jy}^{\text {beam }}{ }^{-1}$. The beam sizes are denoted in the lower left. Middle: the MIPS $70 \mu \mathrm{m}$ map in logarithmic scale. The first two contours are at 130 and $400 \mathrm{MJy} \mathrm{sr}^{-1}$, the following increase in steps of $400 \mathrm{MJy} \mathrm{sr}^{-1}$. The positions of SMM1 and SMM2 derived from interferometric continuum data (see text) are indicated by white crosses. The $F W H M$ of the MIPS $70 \mu \mathrm{m}$ PSF is denoted in the lower left. Right: map of the $24 \mu \mathrm{m}$ emission in logarithmic scale. The three contours at levels of 20,22 and $30 \mathrm{MJy} \mathrm{sr}^{-1}$ are displayed to emphasize the extended emission component. The open squares mark the six emission peaks detected near the cores. The $F W H M$ of the MIPS $24 \mu \mathrm{m}$ PSF is denoted in the lower left.

SMM1 and $F_{24} \mu \mathrm{m}=0.050 \pm 0.004 \mathrm{Jy}$ for SMM2. In addition to the point sources there is also a significant extended emission component. The flux of the entire region is determined to $F_{24 \mu \mathrm{m}}=1.74 \pm 0.09 \mathrm{Jy}$.

\subsection{Dust temperature and masses}

The derived SEDs for the two cores from 24 to $850 \mu \mathrm{m}$ are shown in Fig. 2. The observed flux is due to the thermal emission of dust in this wavelength regime. To determine the temperature of the dust and the total gas mass in the cores, we fitted two modified Planck components to the data. The Planck curves were modified using dust opacity values from Ossenkopf \& Henning (1994) for dust with thick ice mantles $\left(\kappa_{850}=1.0 \mathrm{~cm}^{2} \mathrm{~g}^{-1}\right)$. At long wavelengths, this corresponds to an emissivity index of $\beta \sim 1.8$. For both cores the fit is in very good agreement with the spectrophotometric data.

Using a gas-to-dust ratio of 100 , we find a mass of $M \approx$ $200 M_{\odot}$ for both cores, with the bulk of the material $(>99 \%)$ being very cold $\left(T_{\mathrm{d}}=19.5 \mathrm{~K}\right.$ for SMM1 and $T_{\mathrm{d}}=17.3 \mathrm{~K}$ for SMM2). The derived MIR to millimeter luminosities are $L=1000 L_{\odot}$ and $L=490 L_{\odot}$ for SMM1 and SMM2, respectively. We find $M=900 M_{\odot}$ and $L=2100 L_{\odot}$ for the entire region using the same approach as above and including the IRAS and ISOSS fluxes in the SED fit. The results are summarized in Table 2.

\subsection{Gas masses, temperatures, and densities}

We used our $\mathrm{C}^{18} \mathrm{O}(2-1)$ map to get an independent estimate for the mass of the entire region. Large velocity gradient (LVG) simulations show that for $15 \mathrm{~K} \leq T_{\mathrm{K}} \leq 80 \mathrm{~K}$ and $10^{3} \mathrm{~cm}^{-3} \lesssim$ $n\left(\mathrm{H}_{2}\right) \lesssim 10^{6} \mathrm{~cm}^{-3}$ the dependence of total column density and the intensity of the $\mathrm{C}^{18} \mathrm{O}(2-1)$ line transition takes the simple form (Rohlfs \& Wilson 2004, and references therein) $N_{\mathrm{H}_{2}}\left[\mathrm{~cm}^{-3}\right]=5.3 \times 10^{21} \int T_{\mathrm{MB}}\left(\mathrm{C}^{18} \mathrm{O}, J=2 \rightarrow 1\right) \mathrm{d} v$, assuming that the ratio of $\mathrm{C}^{18} \mathrm{O}$ to $\mathrm{H}_{2}$ is $3.4 \times 10^{-7}$ (Wilson \& Rood 1994).

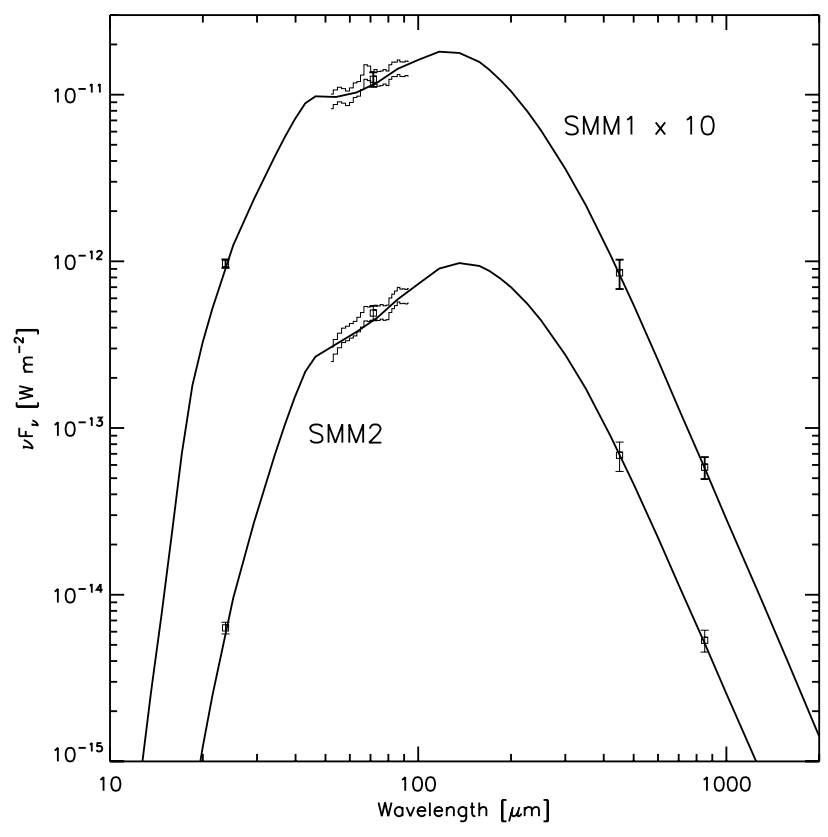

Fig. 2. Spectral energy distribution of SMM1 (multiplied by 10) and SMM2. The square symbols represent photometric data from MIPS at 24 and $70 \mu \mathrm{m}$ and SCUBA at 450 and $850 \mu \mathrm{m}$. The histogram-like bars show the 1-sigma range of our spectrophotometry using the MIPS SED mode (53 to $93 \mu \mathrm{m}$ ). The lines show the combined fitted SEDs for both Planck components, yielding $T_{\text {warm }}=51 \mathrm{~K}$ and $T_{\text {cold }}=19.5 \mathrm{~K}$ for SMM1 and $T_{\text {warm }}=41 \mathrm{~K}$ and $T_{\text {cold }}=17.3 \mathrm{~K}$ for SMM 2 .

The total cloud mass is calculated from our $\mathrm{C}^{18} \mathrm{O}$ data using the relation

$M_{\mathrm{H}_{2}}=\mu m_{\mathrm{H}_{2}} D^{2} \times \sum_{i} \Omega_{i} N_{\mathrm{H}_{2}}^{i}$,

where $m_{\mathrm{H}_{2}}$ is the mass of the hydrogen molecule, $\mu=1.36$ the increase in mass due to other elements (mostly helium), $D$ the distance to the source, and $\Omega_{i}$ the solid angle of the map pixels. We derive a total mass of $M_{\mathrm{H}_{2}}=1000 \pm 200 M_{\odot}$. This is in very 
Table 2. Results of the thermal dust emission modeling.

\begin{tabular}{cccccccc}
\hline \hline Source & $\begin{array}{c}\text { Mass } \\
{\left[M_{\odot}\right]}\end{array}$ & $\begin{array}{c}\text { Luminosity } \\
{\left[L_{\odot}\right]}\end{array}$ & $\begin{array}{c}T_{\text {cold }} \\
{[\mathrm{K}]}\end{array}$ & $\begin{array}{c}T_{\text {warm }}[\mathrm{K}] \\
\end{array}$ & $M_{\text {cold }} / M_{\text {total }}$ & $L_{\text {cold }} / L_{\text {total }}$ & $\begin{array}{c}M / L \\
{\left[M_{\odot} / L_{\odot}\right]}\end{array}$ \\
\hline ISOSS J23053+5953 & 900 & 2100 & 17.1 & 53 & $>0.99$ & 0.72 & 0.43 \\
SMM1 & 195 & 1000 & 19.5 & 51 & $>0.99$ & 0.68 & 0.19 \\
SMM2 & 205 & 490 & 17.3 & 41 & $>0.99$ & 0.76 & 0.42 \\
\hline
\end{tabular}

Table 3. $\mathrm{CH}_{3} \mathrm{OH}$ parameters.

\begin{tabular}{lcc}
\hline \hline Transition & Peak $T_{\mathrm{MB}}[\mathrm{K}]$ & $\int T_{\mathrm{MB}}(v) \mathrm{d} v\left[\mathrm{~K} \mathrm{~km} \mathrm{~s}^{-1}\right]$ \\
\hline $5_{0} \rightarrow 4_{0}-A$ & $1.26 \pm 0.02$ & $8.03 \pm 0.08$ \\
$5_{-1} \rightarrow 4_{-1}-E$ & $1.05 \pm 0.02$ & $6.92 \pm 0.08$ \\
$5_{0} \rightarrow 4_{0}-E$ & $0.29 \pm 0.02$ & $1.42 \pm 0.08$ \\
\hline
\end{tabular}

good agreement with the mass derived from the dust continuum emission.

The low temperatures for the bulk of the material is supported by measurements of the $(J, K)=(1,1)$ and $(2,2)$ inversion transitions of ammonia $\left(\mathrm{NH}_{3}\right)$ by Wouterloot et al. (1988). They found line emission at $v_{\mathrm{LSR}}=-50.8 \mathrm{~km} \mathrm{~s}^{-1}$ and $v_{\mathrm{LSR}}=$ $-52.3 \mathrm{~km} \mathrm{~s}^{-1}$ and gas kinetic temperatures of $T_{\text {kin }}=15.4 \mathrm{~K}$ and $T_{\text {kin }}=14.8 \mathrm{~K}$, respectively, in very good agreement with the dust temperatures derived by us.

We used the measured methanol transition lines to estimate the gas density towards the two cores (Leurini et al. 2004). The relevant lines were observed simultaneously within the same bandpass, therefore uncertainties in the absolute spectrophotometric calibration do not affect the results. The beam size $\left(F W H M \sim 31^{\prime \prime}\right)$ is larger than the separation of the two cores, and the individual spectra have the same line ratios within the measurement errors. The ratio of the observed $-E$ lines is $\log \left[T_{R}\left(5_{0} \rightarrow 4_{0}\right) / T_{R}\left(5_{-1} \rightarrow 4_{-1}\right)\right]=-0.56 \pm 0.04$ (see Table 3). Assuming a methanol abundance of $N\left(\mathrm{CH}_{3} \mathrm{OH}\right) / \Delta v=$ $10^{14} \mathrm{~cm}^{-2}\left(\mathrm{~km} \mathrm{~s}^{-1}\right)^{-1}$, this corresponds to a spatial density of $n\left(\mathrm{H}_{2}\right)=(2 \pm 1) \times 10^{6} \mathrm{~cm}^{-3}$. There were no more methanol lines detected in the observed bandpass (upper limit of $T_{\mathrm{MB}} \leq 0.06 \mathrm{~K}$, 3- $\sigma)$.

Mangum \& Wootten (1993) used a spherical LVG model to show that the two observed formaldehyde transitions are a good tracer for gas kinetic temperatures $T_{\mathrm{K}} \lesssim 50 \mathrm{~K}$ at densities $n\left(\mathrm{H}_{2}\right) \gtrsim 10^{5} \mathrm{~cm}^{-3}$ with only a weak dependence on density. Since both lines can be measured simultaneously within the same bandpass, uncertainties in the absolute spectrophotometric calibration do not affect the results. As for our methanol measurements, the spectra were averaged for both cores as they showed the same line ratios within the measurement uncertainties. The measured line intensities and integrated areas are summarized in Table 4. Using the LVG model of Mangum \& Wootten (1993) with $N\left(\right.$ para $\left.-\mathrm{H}_{2} \mathrm{CO}\right) / \Delta v \approx 10^{13.5} \mathrm{~cm}^{-2}\left(\mathrm{~km} \mathrm{~s}^{-1}\right)^{-1}$, the line intensity ratio of $T_{\mathrm{R}}\left(3_{03} \rightarrow 2_{02}\right) / T_{\mathrm{R}}\left(3_{22} \rightarrow 2_{21}\right)=5.5_{-0.6}^{+0.8}$ leads to a gas kinetic temperature of $T_{\mathrm{K}}=42 \pm 10 \mathrm{~K}$ for a density of $n\left(\mathrm{H}_{2}\right) \approx$ $2 \times 10^{6} \mathrm{~cm}^{-3}$ as inferred from our methanol data. Another temperature estimate was made using the velocity-integrated line ratios assuming local thermal equilibrium (LTE) and optically thin emission. With $\int T_{\mathrm{R}}\left(3_{03} \rightarrow 2_{02}\right) \mathrm{d} v / \int T_{\mathrm{R}}\left(3_{22} \rightarrow 2_{21}\right) \mathrm{d} v=$ $6.9_{-0.7}^{+0.8}$, we find $T_{\mathrm{K}}=35 \pm 3 \mathrm{~K}$, which is lower than the LVG result but well within the uncertainties. Both estimates give temperatures in good agreement with the warm dust components derived in the SED dust modeling. Due to the higher critical density
Table 4. $\mathrm{H}_{2} \mathrm{CO}$ parameters.

\begin{tabular}{ccc}
\hline \hline Transition & Peak $T_{\mathrm{MB}}[\mathrm{K}]$ & $\int T_{\mathrm{MB}}(v) \mathrm{d} v\left[\mathrm{~K} \mathrm{~km} \mathrm{~s}^{-1}\right]$ \\
\hline $3_{03} \rightarrow 2_{02}$ & $0.751 \pm 0.015$ & $4.41 \pm 0.08$ \\
$3_{22} \rightarrow 2_{21}$ & $0.136 \pm 0.015$ & $0.64 \pm 0.06$ \\
\hline
\end{tabular}

of $\mathrm{H}_{2} \mathrm{CO}$ compared to $\mathrm{NH}_{3}$, the observed formaldehyde transitions trace the denser and thus warmer regions of the core.

\subsection{High-resolution mapping}

The PdBI continuum maps are shown in Fig. 3. The beam sizes of $2.58^{\prime \prime} \times 2.16^{\prime \prime}\left(\mathrm{PA}=78^{\circ}\right)$ at $3.4 \mathrm{~mm}$ and $1.15^{\prime \prime} \times 0.87^{\prime \prime}$ $\left(\mathrm{PA}=75^{\circ}\right)$ at $1.3 \mathrm{~mm}$ correspond to approximately $8250 \mathrm{AU}$ and $3500 \mathrm{AU}$, respectively. SMM1 is extended at $3 \mathrm{~mm}$ and resolved into at least two, possibly three, compact objects at $1.3 \mathrm{~mm}$ (SMM1a through 1c). SMM2 appears point-like at $3 \mathrm{~mm}$ and is partly resolved at $1.3 \mathrm{~mm}$, with a point source contributing $11 \mathrm{mJy}$ and a Gaussian-like component with $34 \mathrm{mJy}$ and a $F W H M$ of $2.5^{\prime \prime} \times 1.6^{\prime \prime}\left(\mathrm{PA}=84^{\circ}\right.$, corresponding to $8750 \times$ $5600 \mathrm{AU}$ at a distance of $3.5 \mathrm{kpc}$ ). The derived fluxes for the two cores and their components are summarized in Table 5.

From our SED fit presented in Fig. 2, we determine the fluxes that one would expect for the cores at $1.3 \mathrm{~mm}$ and $3.4 \mathrm{~mm}$, based on the fitted Planck components and the dust opacity values at the wavelengths of interest. These fluxes are also presented in Table 5. The total interferometric flux of SMM1 at $1.3 \mathrm{~mm}$ is only $6.3 \%$ of the flux estimated from single-dish obervations whereas the interferometric flux for SMM2 is $12.5 \%$ expected from a single-dish measurement. This is probably due to the fact that structures larger than $\approx 1 / 4$ of the primary beam size are filtered out by the interferometer (Stanimirovic 2002). With the larger $3 \mathrm{~mm}$ beam about 40 to $60 \%$ of the expected fluxes are recovered. Table 5 also shows the masses of the cores and their components derived from the interferometric $1.3 \mathrm{~mm}$ fluxes, assuming the dust temperatures as found by the fits to the singledish photometry.

\subsection{Gas kinematics}

There is a possible outflow originating from SMM2 detected in the interferometric $\mathrm{CO}(2-1)$ map (see Fig. 3). We derive the outflow mass $M_{\mathrm{f}}=4.6 M_{\odot}$, mechanical momentum $P_{\mathrm{f}}=$ $40 M_{\odot} \mathrm{km} \mathrm{s}^{-1}$, and kinetic energy $E_{\text {kin }}=210 M_{\odot} \mathrm{km}^{2} \mathrm{~s}^{-2}$ using the methods described in Henning et al. (2000a,b), using Eq. (1) with an $\mathrm{H}_{2}$ column density $N\left(\mathrm{H}_{2}\right)\left(\mathrm{cm}^{-2}\right)=3 \times$ $10^{20} \int T_{\mathrm{MB}} \mathrm{d} v$ (Osterloh et al. 1997) and applying a correction factor of $f(i)=2.9$ to account for the effects of the unknown inclination angle $i$ of the outflow (Henning \& Launhardt 1998).

We have obtained single-dish spectra of the $\mathrm{HCO}^{+}(3-2)$ and $\mathrm{H}^{13} \mathrm{CO}^{+}(3-2)$ line towards both cores (Fig. 4). In each case, a red-shifted self absorption profile in the optically thick $\mathrm{HCO}^{+}$line compared to the optically thin $\mathrm{H}^{13} \mathrm{CO}^{+}$line is observed, indicating infall motion of the gas (Choi 2002). The 

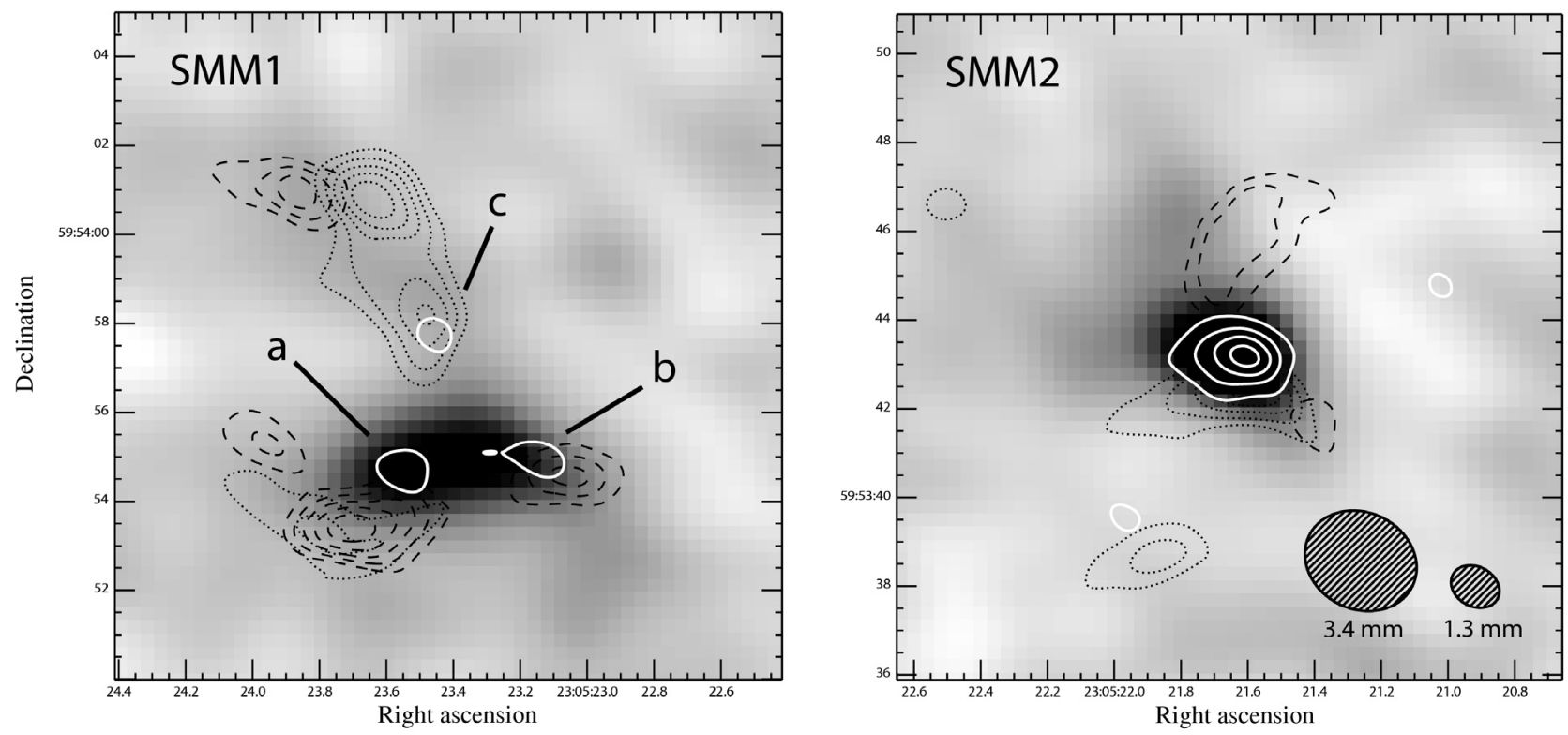

Fig. 3. Interferometric measurements towards ISOSS J23053+5953 SMM1 (left) and SMM2 (right). The linear color scale shows the emission in the $3 \mathrm{~mm}$ continuum with a linear color scale up to $2.6 \mathrm{mJy} / \mathrm{beam}$. The white contours show the $1.3 \mathrm{~mm}$ continuum emission in steps of $3.7 \mathrm{mJy} / \mathrm{beam}\left(5\right.$-sigma). The dashed $\left(v_{\mathrm{LSR}}=-70.5 \ldots-53.5 \mathrm{~km} \mathrm{~s}^{-1}\right)$ and dotted $\left(v_{\mathrm{LSR}}=-48.5 \ldots-32.5 \mathrm{~km} \mathrm{~s}^{-1}\right)$ black contours show the integrated $\mathrm{CO}(2-1)$ line emission starting at $25 \mathrm{~K} \mathrm{~km} \mathrm{~s}^{-1}$ in steps of $15 \mathrm{~K} \mathrm{~km} \mathrm{~s}^{-1}$. The beam sizes are denoted in the lower right.

Table 5. Expected and measured fluxes and masses for the compact components of SMM1 and SMM2.

\begin{tabular}{lcccrr}
\hline \hline & \multicolumn{2}{c}{ Expected flux } & \multicolumn{2}{c}{ Measured flux } & Mass \\
Source & $1.3 \mathrm{~mm} 3.4 \mathrm{~mm}$ & $1.3 \mathrm{~mm} 3.4 \mathrm{~mm}$ & {$\left[M_{\odot}\right]$} \\
& \multicolumn{2}{c}{$[\mathrm{mJy}]$} & \multicolumn{2}{c}{$[\mathrm{mJy}]$} & \\
\hline SMM1 & 400 & 15 & 25 & 8.7 & 12 \\
a & & & 9.0 & & 4.4 \\
b & & & 9.1 & & 4.4 \\
c & & & 5.5 & & 2.7 \\
SMM2 & \multirow{2}{*}{14} & 45 & 5.4 & 26 \\
compact & & & 11 & & 6.5 \\
Gaussian & & & 34 & & 19.5 \\
\hline
\end{tabular}

emission from the two cores is separated with the beam size of approximately $9.5^{\prime \prime}$, suggesting that both sub-mm cores are currently collapsing.

The spectrum of the $\mathrm{HCO}^{+}(1-0)$ line measured with the PdBI towards the compact mm-core SMM2 is presented in Fig. 5, showing a very strong red-shifted self absorption with only the blue part of the line remaining. This suggests infall motion at scales of $\sim 8500 \mathrm{AU}$, the beam size of the $3 \mathrm{~mm}$ measurements. For SMM1, the infall profile observed in the single-dish data is not reproduced in the interferometric $\mathrm{HCO}^{+}(1-0)$ spectra.

\subsection{Active star formation}

The IRAC and NIR images of ISOSS J23053+5953 are presented in Fig. 6. They show several young stellar objects and outflows traced by the emission of shocked molecular hydrogen. Although a detailed classification of all individual YSOs and a characterization of the jets is beyond the scope of this paper, we note the pronounced bipolar jet in the western part of the image that is probably driven by the young stellar object at RA (2000)= $23^{\mathrm{h}} 05^{\mathrm{m}} 18.3$, Dec $\left.(2000)=+59^{\circ} 54^{\prime} 05^{\prime \prime}\right)$. This structure has a projected length of about $2 \mathrm{pc}$ and is observed in the NIR, as well as in the $4.5 \mu \mathrm{m}$ band with IRAC. The images support the

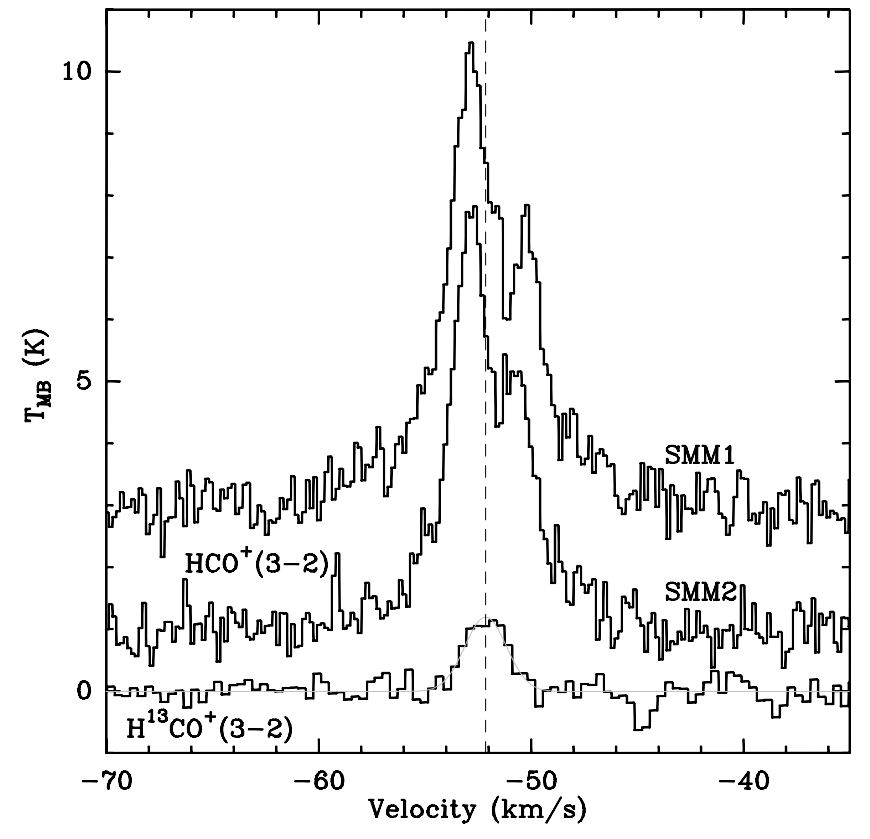

Fig. 4. Spectra of $\mathrm{H}^{(13)} \mathrm{CO}^{+}(3-2)$ measured towards SMM1 and SMM2. The $\mathrm{HCO}^{+}(3-2)$ spectrum of SMM1 and SMM2 were shifted by $3 \mathrm{~K}$ and $1 \mathrm{~K}$, respectively. The grey line is a Gaussian fit to the optically thin $\mathrm{H}^{13} \mathrm{CO}^{+}(3-2)$ emission. The dashed vertical line shows the derived central velocity $v_{\mathrm{LSR}}=-52.127 \pm 0.095 \mathrm{~km} \mathrm{~s}^{-1}$. Both cores show a red-shifted self absorption feature in the optically thick $\mathrm{HCO}^{+}(3-2)$ line with respect to the averaged $\mathrm{H}^{13} \mathrm{CO}^{+}(3-2)$ emission.

finding that SMM1 is more evolved than SMM2 based on the number of YSOs detected in the infrared.

\section{Discussion}

Although a warm $\left(T_{\text {warm }} \sim 50 \mathrm{~K}\right)$ dust compoment is needed to fit the observed $24 \mu \mathrm{m}$ fluxes of ISOSS J23053+5953 SMM1 


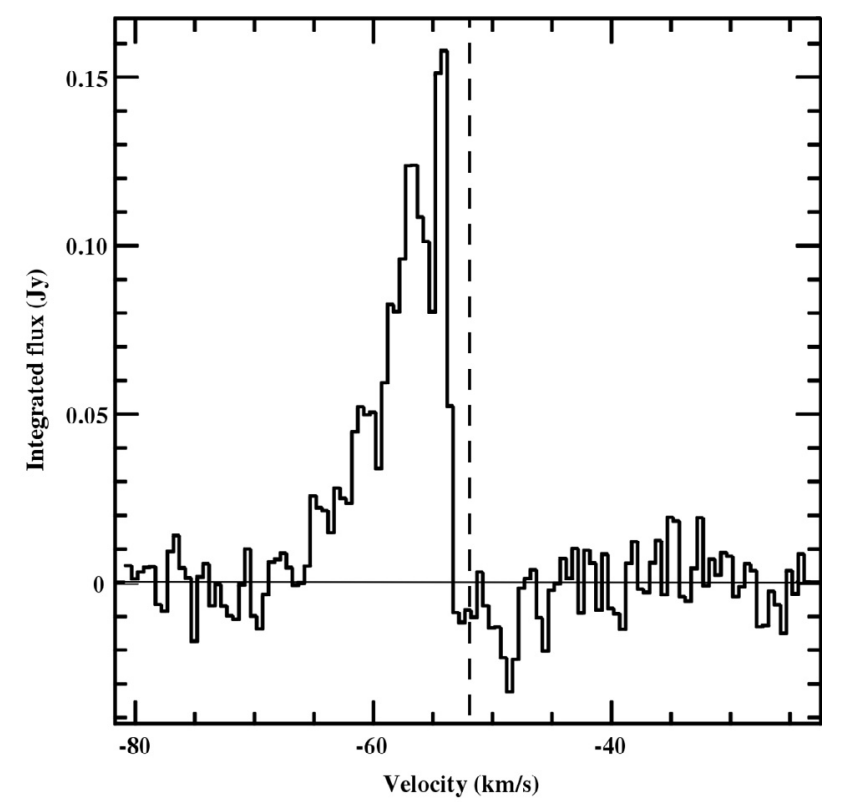

Fig. 5. Spectrum of the $\mathrm{HCO}^{+}(1-0)$ emission line towards SMM2 measured with the PdBI. The vertical dashed line corresponds to the central velocity of optically thin lines.

and SMM2 well, we note that the bulk of the material in the two detected massive $\left(M \sim 200 M_{\odot}\right)$ cores has low $(T<20 \mathrm{~K})$ temperatures. The SEDs contain spectrophotometric data in the FIR with comparable resolution to the sub-mm data. This allows to determine accurate temperatures and masses in contrast to cases where there is no data available in the Wien part of the spectrum.

While both cores show signs of infall in single-dish measurements of $\mathrm{HCO}^{+}(3-2)$, SMM1 is resolved into three compact components with the PdBI in the $1.3 \mathrm{~mm}$ continuum (beam of $\left.\sim 1^{\prime \prime}\right)$. Therefore it is not unambiguous whether the observed line profile is due to infall or to the superposition of several velocity components. The presence of several luminous infrared sources in its vicinity suggests that this core is further evolved and a site of active star formation.

\subsection{The massive protostellar core candidate SMM2}

In contrast to SMM1, the object SMM2 is a promising candidate for an extremely young high-mass protostellar core. It can be described by a compact mm-core plus a Gaussian component with $R_{\text {maj }}=4400 \mathrm{AU}$ and $R_{\min }=2800 \mathrm{AU}$. The mass inside this radius is $M=26 M_{\odot}$. If one assumes an oblate ellipsoid with the given radii as its geometry, this mass results in a spatial density of $n\left(\mathrm{H}_{2}\right)=1.5 \times 10^{7} \mathrm{~cm}^{-3}$. The column density at the peak position $\left(F_{1.3 \mathrm{~mm}}=17 \mathrm{mJy}_{\text {beam }}{ }^{-1}\right)$ is $N\left(\mathrm{H}_{2}\right)=1.3 \times$ $10^{24} \mathrm{~cm}^{-2}$. This core also shows infall in both the single-dish and interferometric line data. The red wing is of the $\mathrm{HCO}^{+}(1-0)$ line emission is fully suppressed, and we interpret this as the result of infalling gas. The much stronger absorption compared to the $\mathrm{HCO}^{+}(3-2)$ line emission is most probably due to the lower critical density of $\mathrm{HCO}^{+}(1-0)$. SMM2 also drives an outflow traced in $\mathrm{CO}(2-1)$ observed with the PdBI. The derived mass and momentum for this outflow are low considering the high mass of the driving source, but one has to take into account that the interferometer filters out large scales and thus does not allow the entire line flux to be reconstructed (Choi et al. 2004).

There is an infrared source detected in the $H, K \mathrm{~s}$, and all four IRAC bands with a separation of $0.4^{\prime \prime}$ to SMM2. Considering the astrometric accuracy of our NIR images $\left(\sim 0.1^{\prime \prime} \mathrm{rms}\right)$ and the PdBI observations $\left(\sim 0.15^{\prime \prime} \mathrm{rms}\right)$, this source is located more than 2- $\sigma$ away from the mm-core but could be the counterpart of SMM2. Assuming an early B-type star, the observed $\mathrm{H}-\mathrm{K}$ color of $2.22 \mathrm{mag}$ implies a visual extinction of $A_{\mathrm{V}} \approx 37 \mathrm{mag}$ (Rieke \& Lebofsky 1985). The dereddened SED is well-matched by a Kurucz (1979) model for a B2.5V star $\left(T_{\text {eff }}=20000 \mathrm{~K}\right.$, $\log (g)=4.0)$ at a distance of $3.5 \mathrm{kpc}$, apart from excess emission observed in the 5.8 and $8 \mu \mathrm{m}$ IRAC bands.

The column density of $N\left(\mathrm{H}_{2}\right)=1.3 \times 10^{24} \mathrm{~cm}^{-2}$ measured towards SMM2, however, corresponds to an extinction of $A_{\mathrm{V}} \sim$ $350 \mathrm{mag}$ in the center of the core, if one assumes a spherical density distribution and a dust-to-gas ratio of $N_{\mathrm{H}} / A_{\mathrm{V}}=1.87 \times$ $10^{21} \mathrm{~cm}^{-2} \mathrm{mag}^{-1}$ (Savage \& Mathis 1979). The derived extinction of only $A_{\mathrm{V}}=37.1$ mag would then require a donut or disklike gas and dust distribution, with a relatively free cone that is seen towards the center of the core. Yet a B2.5V star would have a luminosity of $L \sim 2100 L_{\odot}$ (de Jager \& Nieuwenhuijzen 1987), inconsistent with the observed MIR-to-mm luminosity of SMM2.

Another explanation for the observed MIR excess of the counterpart candidate would be the flux originating in a disk around a T Tauri like object. Using the relationships of Carpenter et al. (1993), $\log (M)=-0.24 M_{\mathrm{K}}+0.24$, and $\log (M)=$ $-0.25 M_{\mathrm{H}}+0.44$, we derived an extinction of $A_{\mathrm{V}} \sim 23$ mag and a mass of $M \sim 1.2 M_{\odot}$. Such a YSO would have a luminosity of $L \lesssim 6 L_{\odot}$ (Calvet et al. 2004) and cannot explain the observed luminosity of $480 L_{\odot}$ of SMM2.

We therefore conclude (i) that the NIR source near the center of SMM2 is not the counterpart of a central object, but rather a low- to intermediate mass YSO located at the outer rim of the mm-core, and (ii) that its vicinity to the latter one is only a projection effect. But if the observed luminosity is not generated by a main sequence star or an evolved YSO, what is its origin? Possibilities are external heating from the interstellar radiation field and the nearby YSOs, accretion luminosity of an embedded protostar, or a combination of both. The observed blue-skewed $\mathrm{HCO}^{+}$spectra indicate infall motion towards the core center. During the early phases, the total luminosity of an emerging protostar is mainly due to accretion and depends on the accretion rate $\dot{M}_{*}$ (Palla \& Stahler 1992). In particular, the accretion luminosity is

$L_{\mathrm{acc}}=\frac{f_{\mathrm{acc}} G M_{*}(t) \dot{M}_{*}(t)}{R_{*}(t)}$

where $G$ is the gravitational constant, $M_{*}$ the mass of the protostar, $R_{*}$ its radius, $\dot{M}_{*}$ the accretion rate, and $f_{\text {acc }} \approx 0.5$ a factor that accounts for energy used to drive an outflow. However, the mass of the protostar, its radius, and the accretion rate are all time dependent. Using the models for massive protostar evolution by McKee \& Tan (2003), we find that the observed luminosity, core mass, and surface density $\Sigma \approx 6 \mathrm{~g} \mathrm{~cm}^{-2}$ correspond to a massive molecular core with an emerging protostar.

To further characterize the protostellar core and its central source, we compared the observed SED with the results of twodimensional radiative transfer simulations by Robitaille et al. (2006). They recently used the Monte-Carlo-code by Whitney et al. (2003) to compute the SEDs for more than 20000 young stellar objects at different inclinations of the disk and envelope cavity. The measured energy distribution of SMM2 only fits the model with the ID 3000552 resonably well (see Fig. 7). According to this model, the central source is a very young $(t \sim 5000 \mathrm{yr})$ and accreting protostar with an actual mass of 


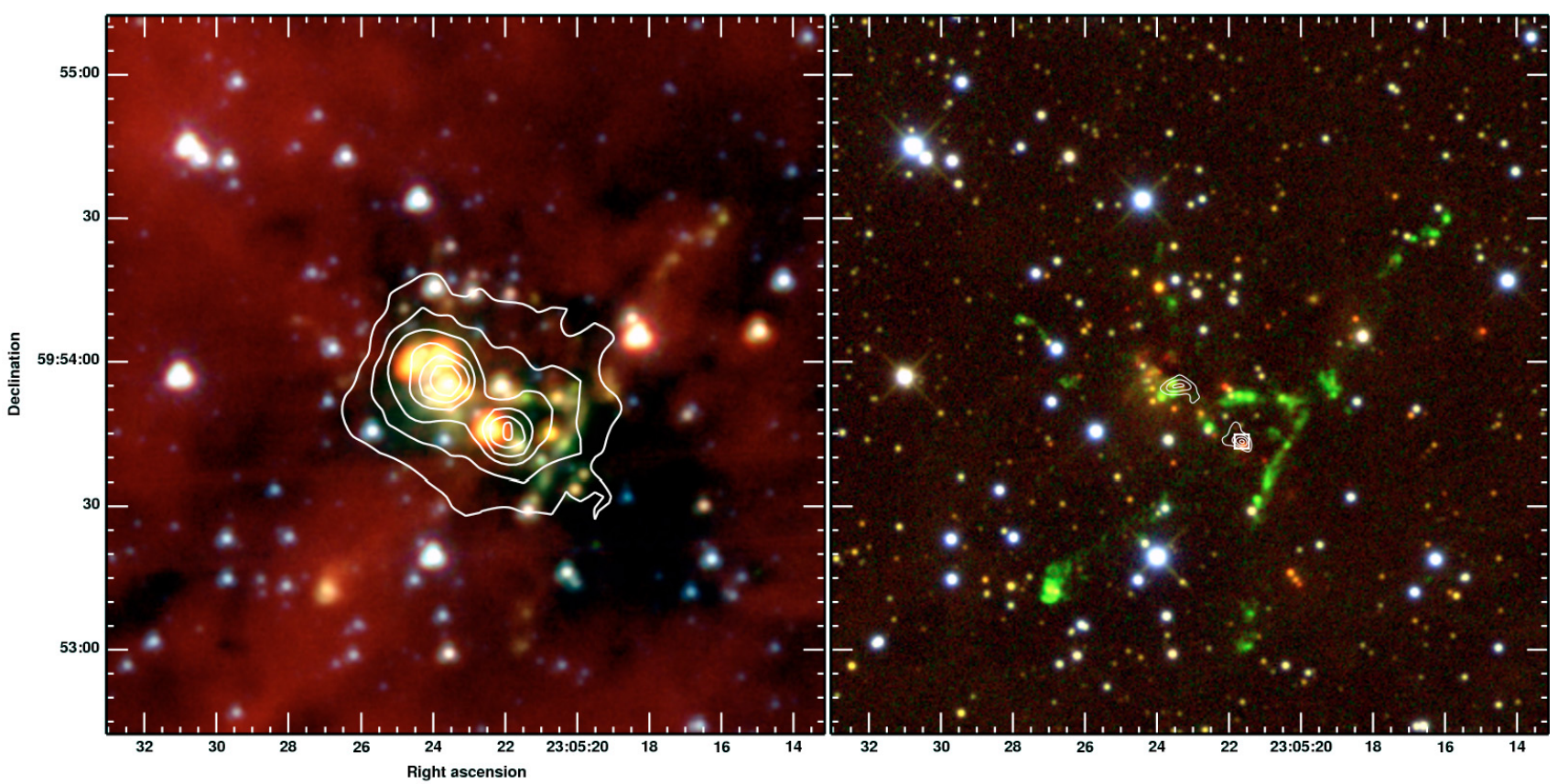

Fig. 6. Left: color composite image created from the four IRAC channels with 3.6, 4.5, 5.8, and $8 \mu \mathrm{m}$ mapped to blue, green, orange, and red, respectively. The white contours show the $450 \mu \mathrm{m}$ continuum emission as in Fig. 1. Right: color composite image created from our near-infrared data with $J, H, K \mathrm{~s}$, and $\mathrm{H}_{2}(\lambda=2.122 \mu \mathrm{m})$ mapped to blue, green, red, and green. The white box denotes the position of SMM2 as derived from PdBI interferometry, and the white contours show the $3 \mathrm{~mm}$ continuum in steps of $0.6 \mathrm{mJy} / \mathrm{beam}$.

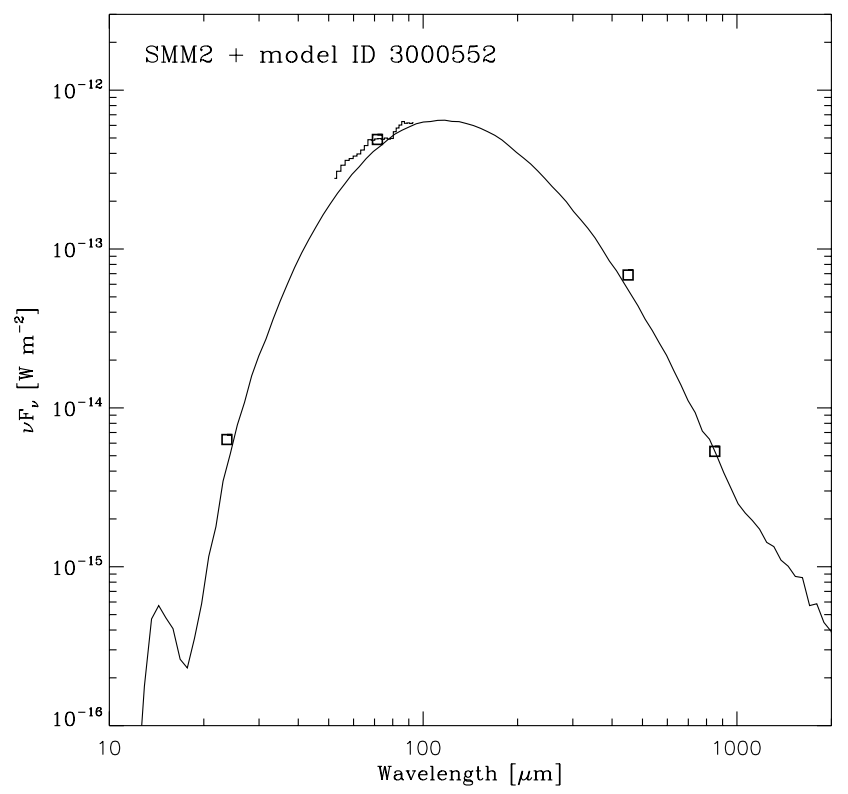

Fig. 7. SED of SMM2 (squares and stepped line) and of the model ID 3000552 from Robitaille et al. (2006) for an inclination of $i \approx 32^{\circ}$ (solid line).

$M_{*} \sim 4.6 M_{\odot}$, a radius of $R_{*} \sim 33 R_{\odot}$, and effective surface temperature of $T_{\text {eff }} \sim 4200 \mathrm{~K}$. The infall rate of the envelope is $\dot{M}_{\text {core }}=2.1 \times 10^{-3} M_{\odot} \mathrm{yr}^{-1}$ and high enough to suggest the further growth of the central source. The infall rate is about a factor of two higher than the observed outflow rate, if one assumes a dynamical flow age of $t_{\text {dyn }} \approx 5000 \mathrm{yr}$, equal to the age of the protostar for the best fitting model.

To assess the quality of this result, we compared the derived parameters to those of the ten next best-fitting models. In all cases the mass of the protostar was $M_{*} \geq 4 M_{\odot}$ and the age less than $3.6 \times 10^{4} \mathrm{yr}$. This supports the finding that SMM2 is indeed a very young protostellar core.

\section{Summary}

We have identified a massive protostellar core in the star-forming region ISOSS J23053+5953 using ground and Spitzer based follow-up observations. This core has a mass of $M \sim 200 M_{\odot}$ and a temperature of $T \sim 17 \mathrm{~K}$ and shows signs of infall in both single-dish and interferometric measurements. The luminosity of this object $\left(L=490 L_{\odot}\right)$ and its SED can be explained by the presence of a very young accreting protostar embedded in a massive envelope.

Acknowledgements. Based on observations with ISO, an ESA project with instruments funded by ESA Member States (especially the PI countries: France, Germany, the Netherlands, and the United Kingdom) and with the participation of ISAS and NASA. The ISOSS is supported by funds from the DLR, Bonn. Based on observations collected at the Centro Astronómico Hispano Alemán (CAHA) at Calar Alto, operated jointly by the Max-Planck Institut für Astronomie and the Instituto de Astrofísica de Andalucía (CSIC). Based on observations carried out with the IRAM 30m and the IRAM Plateau de Bure Interferometer. IRAM is supported by INSU/CNRS (France), MPG (Germany), and IGN (Spain). Based on observations with the James-ClerkMaxwell Telescope (JCMT) and the Sub-Millimeter-Telescope (SMT, formerly the Heinrich-Hertz-Telescope, HHT). This work is based in part on observations made with the Spitzer Space Telescope, which is operated by the Jet Propulsion Laboratory, California Institute of Technology under a contract with NASA. We acknowledge the support of P. Salome during PdBI data reduction at the IRAM office, Grenoble.

\section{References}

Baumeister, H., Bizenberger, P., Bayler-Jones, C. A. L., et al. 2003, in Instrument Design and Performance for Optical/Infrared Ground-based Telescopes, ed. M. Iye, \& A. F. M. Moorwood, Proc. SPIE, 4841, 343

Beltrán, M. T., Cesaroni, R., Codella, C., et al. 2006, Nature, 443, 427

Beuther, H., \& Steinacker, J. 2007, ArXiv Astrophysics e-prints

Beuther, H., Sridharan, T. K., \& Saito, M. 2005, ApJ, 634, L185 
Beuther, H., Zhang, Q., Sridharan, T. K., Lee, C.-F., \& Zapata, L. A. 2006, A\&A, 454, 221

Birkmann, S. M., Krause, O., \& Lemke, D. 2006, ApJ, 637, 380

Bizenberger, P., McCaughrean, M. J., Birk, C., Thompson, D., \& Storz, C. 1998, in Infrared Astronomical Instrumentation, ed. A. M. Fowler, Proc. SPIE, 3354, 825

Bogun, S., Lemke, D., Klaas, U., et al. 1996, A\&A, 315, L71

Calvet, N., Muzerolle, J., Briceño, C., et al. 2004, AJ, 128, 1294

Carpenter, J. M., Snell, R. L., Schloerb, F. P., \& Skrutskie, M. F. 1993, ApJ, 407, 657

Choi, M. 2002, ApJ, 575, 900

Choi, M., Kamazaki, T., Tatematsu, K., \& Panis, J.-F. 2004, ApJ, 617, 1157

de Jager, C., \& Nieuwenhuijzen, H. 1987, A\&A, 177, 217

Fazio, G. G., Hora, J. L., Allen, L. E., et al. 2004, ApJS, 154, 10

Forbrich, J., Schreyer, K., Posselt, B., Klein, R., \& Henning, T. 2004, ApJ, 602, 843

Garay, G., Faúndez, S., Mardones, D., et al. 2004, ApJ, 610, 313

Henning, T., \& Launhardt, R. 1998, A\&A, 338, 223

Henning, T., Lapinov, A., Schreyer, K., Stecklum, B., \& Zinchenko, I. 2000a, A\&A, 364, 613

Henning, T., Schreyer, K., Launhardt, R., \& Burkert, A. 2000b, A\&A, 353, 211

Holland, W. S., Robson, E. I., Gear, W. K., et al. 1999, MNRAS, 303, 659

Kessler, M. F., Steinz, J. A., Anderegg, M. E., et al. 1996, A\&A, 315, L27

Keto, E., \& Wood, K. 2006, ApJ, 637, 850

Krause, O. 2003, Ph.D. Thesis

Krause, O., Lemke, D., Tóth, L. V., et al. 2003, A\&A, 398, 1007

Kurucz, R. L. 1979, ApJS, 40, 1

Lemke, D., Klaas, U., Abolins, J., et al. 1996, A\&A, 315, L64

Leurini, S., Schilke, P., Menten, K. M., et al. 2004, A\&A, 422, 573
Low, F. J., Smith, P. S., Werner, M., et al. 2005, ApJ, 631, 1170

Makovoz, D., \& Marleau, F. R. 2005, PASP, 117, 1113

Mangum, J. G., \& Wootten, A. 1993, ApJS, 89, 123

McKee, C. F., \& Tan, J. C. 2003, ApJ, 585, 850

Ossenkopf, V., \& Henning, T. 1994, A\&A, 291, 943

Osterloh, M., Henning, T., \& Launhardt, R. 1997, ApJS, 110, 71

Palla, F., \& Stahler, S. W. 1992, ApJ, 392, 667

Puga, E., Feldt, M., Alvarez, C., et al. 2006, ApJ, 641, 373

Rieke, G. H., \& Lebofsky, M. J. 1985, ApJ, 288, 618

Rieke, G. H., Young, E. T., Engelbracht, C. W., et al. 2004, ApJS, 154, 25

Robitaille, T. P., Whitney, B. A., Indebetouw, R., Wood, K., \& Denzmore, P. 2006, ApJS, 167, 256

Rohlfs, K., \& Wilson, T. L. 2004, Tools of radio astronomy, 4th rev. and enl., ed. K. Rohlfs, \& T. L. Wilson (Berlin: Springer)

Sandell, G., \& Weintraub, D. A. 2001, ApJS, 134, 115

Savage, B. D., \& Mathis, J. S. 1979, ARA\&A, 17, 73

Schuster, K.-F., Boucher, C., Brunswig, W., et al. 2004, A\&A, 423, 1171

Stanimirovic, S. 2002, in Single-Dish Radio Astronomy: Techniques and Applications, ed. S. Stanimirovic, D. Altschuler, P. Goldsmith, \& C. Salter, ASP Conf. Ser., 278, 375

Steinacker, J., Chini, R., Nielbock, M., et al. 2006, A\&A, 456, 1013

Stickel, M., Lemke, D., Klaas, U., Krause, O., \& Egner, S. 2004, A\&A, 422, 39

Ward-Thompson, D. 2002, Science, 295, 76

Werner, M. W., Roellig, T. L., Low, F. J., et al. 2004, ApJS, 154, 1

Whitney, B. A., Wood, K., Bjorkman, J. E., \& Cohen, M. 2003, ApJ, 598, 1079

Wilson, T. L., \& Rood, R. 1994, ARA\&A, 32, 191

Wouterloot, J. G. A., Walmsley, C. M., \& Henkel, C. 1988, A\&A, 203, 367

Wouterloot, J. G. A., Henkel, C., \& Walmsley, C. M. 1989, A\&A, 215, 131

Wu, Y., Zhu, M., Wei, Y., et al. 2005, ApJ, 628, L57 